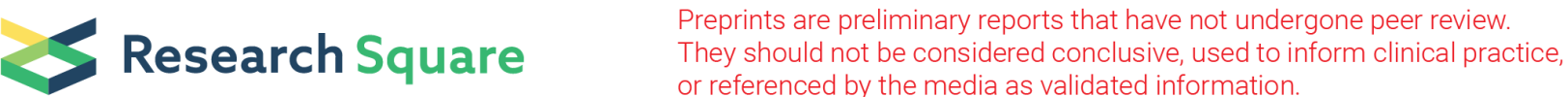

\section{Functional near-infrared spectroscopy combined with unilateral task-oriented training with and without an orthosis in subacute stroke: A pilot study}

Chao Jin Zi Li

School of Rehabilitation, Capital Medical University https://orcid.org/0000-0001-8067-8492

\section{Yih Wong}

Department of Research, Sunnaas Rehabilitation Hospital https://orcid.org/0000-0002-9193-0160

\section{Birgitta Langhammer}

Department of Research, Sunnaas Rehabilitation Hospital https://orcid.org/0000-0002-9639-0570

\section{Fu Biao Huang}

Department of Occupational therapy, Beijing Bo'ai Hospital, China Rehabilitation Research Center https://orcid.org/0000-0002-7686-5153

\section{Xiao Xia Du}

School of Rehabilitation, Capital Medical University https://orcid.org/0000-0001-7944-2474

\section{Yun Lei Wang}

School of Rehabilitation, Capital Medical University https://orcid.org/0000-0001-9954-6246

Hao Jie Zhang

School of Rehabilitation, Capital Medical University https://orcid.org/0000-0001-6889-0853

\section{Tong Zhang ( $\square$ tom611@126.com)}

School of Rehabilitation, Capital Medical University https://orcid.org/0000-0003-0898-1766

\section{Research Article}

Keywords: subacute stroke, rehabilitation, fNIRS, laterality index, upper extremity

Posted Date: August 12th, 2021

DOI: https://doi.org/10.21203/rs.3.rs-806290/v1

License: (c) (i) This work is licensed under a Creative Commons Attribution 4.0 International License. Read Full License 


\section{Abstract \\ Background}

The recovery of upper extremity motor deficits represents a functional challenge for persons with stroke. The aim of this study was to evaluate the mechanisms of Unilateral task-oriented training combined with dynamic hand orthosis for the recovery in subacute stroke by using functional near-infrared spectroscopy (fNIRS).

\section{Methods}

This was a pilot randomized, controlled, assessor-blinded trial. A total sample of 30 subacute persons with stroke were randomized into two groups: the orthosis group and the usual exercise group. All participants received four weeks (60 min/day, 5 days/week) of unilateral upper limb training. The orthosis group wore a dynamic hand orthosis during half the training ( $30 \mathrm{~min} /$ day). $02 \mathrm{Hb}$ concentrations and a laterality index for bilateral premotor cortex, sensorimotor cortex by fNIRS were calculated as outcome indicators that were measured at baseline and 4 weeks after the interventions. Clinical outcomes were collected for correlation analysis.

\section{Results}

No significant difference in $\mathrm{O}_{2} \mathrm{Hb}$ concentrations change between two groups. Cortical activation shifted from the ipsilateral to the contralateral premotor cortex and from the contralateral to the ipsilateral sensorimotor cortex in both groups. In the Orthosis group, the change of Fugl-Meyer Assessment of arm score was positively correlated to the shift to contralateral premotor cortex activation and the change of Action Research Arm Test score was positively correlated to the shift to ipsilateral sensorimotor cortex activation. In the UL group, a negative correlation was observed between the shift to ipsilateral sensorimotor cortex and changes of Fugl-Meyer Assessment of arm score.

\section{Conclusion}

This is the first fNIRS study to explore the mechanism of recovery in subacute stroke after utilizing unilateral task-oriented training and a dynamic orthosis. The current results suggest that fNIRS combined with clinical tests may be useful to evaluate the mechanism of stroke recovery after different rehabilitation interventions. Trial registration: Registered on January 11, 2018 at ClinicalTrials.gov (NCT03396939). https://clinicaltrials.gov/ct2/home.

\section{Background}


Persons with stroke may experience a high rate of disability $(1,2)$. In the acute stage of stroke, two-thirds may experience upper limb and hand dysfunction and three months after stroke onset, $50 \%$ still experience disability in regard to arm-hand performance, such as loss of fine motor control and deficits in motor planning and sensorimotor integration(3).Many persons with stroke, $30 \%-66 \%$, experience reduced motor function in the upper extremity 6 months after debut(4). The upper extremity functions are important for independence in activities of daily living and for perceived quality of life(5).Spontaneous recovery, defined by Bernhardt as the amount of improvement in body function and activity when the recovery of behavior is improved in the absence of a specific, targeted treatment and occurs during a time-sensitive window(6). The subacute stroke phase which may be defined as an early subacute phase(7 days-3 months)and a late subacute phase(3-6 months) since stroke onset (6).The recovery in combination with targeted treatment tends to occur within the first 3 months after stroke onset $(7,8)$. Interpretations in stroke rehabilitation depend on the elucidation of both spontaneous and therapeuticdriven mechanisms of recovery (8).Through either repairment of impairment (regaining movement patterns before stroke), or through compensation (using alternative movements, methods to accomplish the same goal), functional recovery can occur. Rapid establishment of independence in activities of daily living through compensatory strategies is emphasized in current neurorehabilitation practice(9).Thus, rehabilitation training is suggested initiating early to enhance and direct spontaneous recovery. Furthermore, high-quality evidence related to the effectiveness and mechanism of interventions to improve upper limb function is urgently needed(10).

The application of a dynamic hand orthosis combined with task-oriented training has been reported during subacute stage in case studies (11) (12) (13). These moderate-to-severe stroke persons may display small or modest improvements and appear to benefit from training with a dynamic arm orthosis(13). However, studies on the possible neuroplastic mechanism based on task-oriented training methods combined with assistive devices for subacute persons with stroke and particularly randomized controlled trials (RCT) remain scarce (14) (15).Functional near-infrared spectroscopy (fNIRS) can be used to monitor the change of direct and indirect brain regions-regions of interest (ROI)(16) involved in complex motor learning(17) to reflect cerebral cortical reorganization during the process of stroke recovery(18) (19) (20) (21).fNIRS has many advantages, including non-invasiveness, low-cost, portability, and ease of operation(22) (23), that make it suitable for studying the cortical response to simple or complex motor stimulation.

Premotor cortex (PMC) is defined as the anterior half of the precentral gyrus and the anterior bank of the precentral sulcus and the sensorimotor cortex (SMC) is defined as the combination of primary motor and primary sensory. We selected these two area as ROI which plays an important role in functional recovery after motor impairment. Therefore, the aim of this study was to determine the clinical effectiveness of the dynamic hand orthosis in the early subacute stroke period and explore the recovery mechanism with lateralization and oxygenated hemoglobin changes based on motor-task related regions of interest by use of fNIRS in subacute persons with stroke in this paper. 


\section{Methods}

\section{Design}

A Randomized, controlled trial.

\section{Subjects}

A total of 30 persons with stroke were recruited from the neurorehabilitation ward in the China Rehabilitation Research Center (CRRC) from August 2019 to February 2020. The participants were divided into two groups: one unilateral task-oriented training combined with a dynamic hand orthosis group (Orthosis Group) and a usual exercise group (UL group), with 15 persons with strokes in each group.

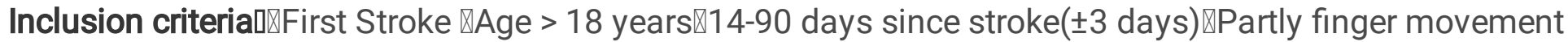

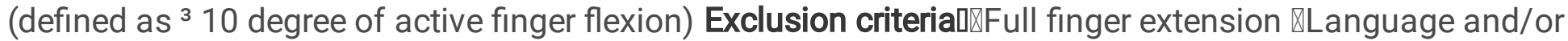
cognitive impairments that preclude the person from following instructions (defined as Mini-Mental State Examination, MMSE $\leq 20$ score『Other health conditions that preclude the person from undergoing rehabilitation, such as severe depression, anxiety, mental symptoms, internal disease(Fig. 1).

\section{Randomization and blinding}

A computer-generated randomization number table was generated in Excel and maintained off-site. Group allocations were distributed in opaque envelopes. To ensure data reliability, the clinical scale and fNIRS measurements were blinded to the testers.

\section{Interventions: unilateral task-oriented training (UL practice) with And without an orthosis}

All the persons with stroke performed task-oriented therapy for the affected upper extremity, for 5 times/week, for 4 consecutive weeks. Individual therapist-supervised upper limb practice could be divided into smaller sessions throughout the training but was set to be 60 minutes in total for arm and hand. The 60 minute session was divided into 10 minutes gross motor training, 10 minutes fine motor training, 10 minutes intensive training, and 30 minutes of activities of daily living training. The Orthosis group wore a hand orthosis device on the affected hand during the supervised UL practice for 30 minutes. The Usual Care group performed supervised UL practice without orthosis for 60 minutes. All the persons with stroke were under the supervision of a licensed OT therapist in both groups. The position of training was individually adapted and performed either in sitting or standing.

The dynamic hand orthosis (Saeboglove ${ }^{\circledR}$, Saebo Inc, Charlotte, NC) used in the study is a hand device equipped with a proprietary tension system that extends the persons' fingers and thumb when grasping. The orthosis was used during repetitive task training, including constant grip-release actions. Releasing is facilitated through finger extension just after the grasping movement has ended ${ }^{(13)}$.The support provided 
to hand opening can be adjusted by the therapist or the persons themselves, depending on the amount of assistance required to accomplish tasks.

\section{Clinical outcome measures}

Clinical outcome measures included the Grip Strength Evaluation (Jamar Digital Hand Dynamometer, $\mathrm{kg}$ ), the Action Research Arm Test (ARAT) ${ }^{(13)}$ and the Fugl-Meyer Assessment of the arm (FMA-arm) ${ }^{(24)}$, were evaluated at the time of recruitment and 4 weeks of unilateral task-oriented training with and without an orthosis.

\section{fNIRS experimental procedure}

fNIRS measurements were performed at the same day of recruitment and the day after 4 weeks of intervention with the subjects in an upright, sitting position, with the eyes closed. Both upper limbs were placed on the knees, with proximal and trunk relaxed. The subject was instructed to grip-release a 9.06kilogram grip ring with the hemiplegic hand. The experiment used a block design with 5 repeated cycles of $30 \mathrm{~s}$ rest and $15 \mathrm{~s}$ movement. The experiment started with a $10 \mathrm{~s}$ pre-scan and a $30 \mathrm{~s}$ rest time, which were not used for statistical analysis. The participant was instructed to repeatedly grip-release with the hemiplegic hand 5 times at a steady speed, guided by an auditory metronome during the 15 second period. The task required the participant to relax the non-grasping hand and to avoid any movements other than those required for the motor tasks during the performance of the one-handed grip-release task. Activation and relaxation were monitored and recorded by the tester.

\section{Data acquisition}

The optical signal was measured using a 48-channel near-infrared spectroscopy (NIRS) machine (Hitachi, ETG-4100). Near-infrared lights with wavelengths of $695 \mathrm{~nm}$ and $830 \mathrm{~nm}$ were guided by optical fiber bundles and transmitted into the brain through the cranium to measure changes in the oxyhemoglobin $\left(\mathrm{O}_{2} \mathrm{Hb}\right)$ and deoxyhemoglobin $\left(\mathrm{de}-\mathrm{O}_{2} \mathrm{Hb}\right)$ concentration at a sampling frequency of $10 \mathrm{~Hz}$ during the motor task. Two plastic probe holders ( $4 \times 4$ matrix) with 24 channels positioned on either side of the head were placed on the scalp over the persons' bilateral motor-related areas. The source-detector probe geometry of the fNIRS system is shown in Fig.1B. A total of 8 sources and 8 detector fiber bundles were positioned on each plastic probe holder. The probes were placed $3 \mathrm{~cm}$ away from each other, to monitor the cortical activation over two $9 \mathrm{~cm} \times 9 \mathrm{~cm}$ rectangular fields of view. The electroencephalography (EEG) International 10-20 system Cz, C3, C4 anatomical measurements were used as reference points to ensure that the optical probe setup was placed over 6 ROls (25), including the bilateral SMC, PMC and PFC. A three-dimensional (3D)-digitizer was used to record the exact locations of each fNIRS probe for a 
standard brain before converting these coordinates into the locations of the forty-eight channels in an estimated Montreal Neurological Institute (MNI) space using the MATLAB toolbox NIRS-SPM (26). The positioning of the 48 channels on a reconstructed 3D brain is shown in Fig.2A (20). Based on the mean MNI coordinates and Brodmann's area (BA) correspondences, the 6 ROls were covered by the following channels for the left and the right hemispheres: the left SMC was covered by channels 4, 5, 6, 8, and 9 (both sides of C3); the right SMC was covered by channels 25, 29, 32, 36, and 39 (both sides of C4); the left PMC was covered by channels $11,12,13,15$, and 16 ; the right PMC was covered by channels 26,30 , 33,37 , and 40 ; the left PFC was covered by channels $18,19,20,22$, and 23 ; and the right PFC was covered by channels $27,31,34,38$, and 41 (Fig.2B).

\section{fNIRS data processing and image analysis}

All artifact data were autodetected and corrected by the open-source HOMER software, implemented in MATLAB(27).Any data with obvious motion artifacts or damaged channels in any block/rest period were manually excluded from further analysis. Then, a $0.01-0.1 \mathrm{~Hz}$ bandpass filter was applied to remove global fluctuation due to heartbeat $(0.8-2.0 \mathrm{~Hz})$, respiration $(0.1-0.33 \mathrm{~Hz})$, and Mayer waves $(0.1 \mathrm{~Hz}$ or lower) (28) (29).

\section{fNIRS outcome measures}

\section{Oxygenated hemoglobin changes based on SMC and PMC}

After preliminary data processing as above, fNIRS was used to classify motor-related brain activity on the sensor-level, and the original light intensity data was converted into a change in the blood oxygen concentration. The average change in $\mathrm{O}_{2} \mathrm{Hb}$ concentration during the movement period and the rest period during each of the five blocks were statistically analyzed. Because fNIRS relies on hemodynamic responses, which take some time to occur, $\mathrm{O}_{2} \mathrm{Hb}$ and de- $\mathrm{O}_{2} \mathrm{Hb}$ signals are typically considered to mark changes in neural activity with a lag of roughly four seconds $₫ 10-14 \mathrm{~s} \rrbracket(30)$. Thus the data of 10 second to 15 second since each block begun were statistically analyzed finally. We used a task-related increase in $\mathrm{O}_{2} \mathrm{Hb}$ concentration value as the marker for cortical activity(31).

\section{Calculating the lateralization index (LI)}

Hemispheric dominance during a motor task is usually expressed for an ROI by calculating the lateralization index. The LI was determined from the sum of oxy-hemoglobin concentrations in channels in each $\mathrm{ROI}$ as $\mathrm{LI}=$ (contralateral $\mathrm{O}_{2} \mathrm{Hb}$ concentration - ipsilateral $\mathrm{O}_{2} \mathrm{Hb}$ concentration)/ (contralateral $\mathrm{O}_{2} \mathrm{Hb}$ concentration + ipsilateral $\mathrm{O}_{2} \mathrm{Hb}$ concentration) (20) (32). LI values range from -1 to 1 , with a score of 1 indicating a purely contralateral ROI (contralateral hemisphere of the tested hand $\mathbb{Z}$ 
affected hemisphere) and -1 indicating a purely ipsilateral ROI (ipsilateral hemisphere of the tested hand囚unaffected hemisphere) activation. The change of $\mathrm{LI}$ values for $\mathrm{PMC}$ and $\mathrm{SMC}=(\mathrm{LI}$ values postintervention) - (LI values pre- intervention).

\section{Statistical analysis}

All statistical analyses were performed using SPSS 19.0. The clinical characteristics (age, gender, time since stroke onset, stroke type, amount of therapy, side of hemiplegia) were compared between the two groups. Measurement data conforming to normal distributions are presented as the mean \pm standard deviation (mean $\pm S D$ ), and within-group comparisons were performed using paired t-tests. Measurement data with non-normal distribution were compared with the rank-sum test. Measurements between the two groups for continuous variables were compared with a two-sample t-test or Mann-Whitney U test. Between group continuous data were analyzed with Chi-square test. Pearson's correlation coefficient was used to analyze the correlation between the change of $\mathrm{LI}$ and clinical scales.LI data are presented as the mean $\pm S D$. The change of $L I$ values for PMC and SMC between group was analyzed with two independent samples t-test. Analysis of changes in $\mathrm{O}_{2} \mathrm{Hb}$ levels in SMC and PFC: Paired t-test was used to compare before and after the intervention, and an independent-samples t-test was used for comparisons between the two groups. Significance was set at $p<0.05$. The confidence interval was $95 \%$.

\section{Sample size and statistical power}

Based on the prior feasibility study from Yih, ipsilesional ARAT scores increased by $7.3 \pm 3.3$ from the first clinical scale assessment ( 0 week) to the second clinical scale assessment (12 week), If a paired sample design is assumed, to achieve $80 \%$ power of test, at alpha $=0.05,1-\beta=0.8, S D=1.96 \times 7.3=14.308$, a total of 33 sample size in each group will achieve. Considering that the sample size is consistent with recommendations in the design of pilot studies, a sample of 15 cases was recruited in each group.

\section{Results}

\section{Characteristics of persons with stroke}

There were no dropouts. No significant differences were observed between the two groups for age, gender, time since stroke onset, stroke type, amount of therapy, or hemiplegia side (Table 1, p>0.05). 


\begin{tabular}{|c|c|c|c|}
\hline characteristic & Orthosis group & UL group & p-value \\
\hline $\mathrm{N}$ & 15 & 15 & \\
\hline Age, years, mean $\pm S D$ & $62.67 \pm 8.95$ & $55.80 \pm 12.42$ & 0.093 \\
\hline \multicolumn{4}{|l|}{ Gender, $\mathrm{n}(\%)$} \\
\hline Male & $10 \rrbracket 66.67 \% \rrbracket$ & $13 \llbracket 86.67 \% \rrbracket$ & 0.195 \\
\hline Female & $5 \rrbracket 33.33 \% \rrbracket$ & $2 \rrbracket 13.33 \% \rrbracket$ & \\
\hline Time since onset, days, mean $\pm S D$ & $53.66 \pm 25.64$ & $51.20 \pm 24.94$ & 0.818 \\
\hline \multicolumn{4}{|l|}{ Stroke type ,n(\%) } \\
\hline Hemorrhage & $6 \rrbracket 40.00 \% \rrbracket$ & $5 \otimes 33.33 \% \bigotimes$ & 0.705 \\
\hline Infarction & $9 \llbracket 60.00 \% \rrbracket$ & $10 \rrbracket 66.67 \% \rrbracket$ & \\
\hline Amount of therapy, hours, mean $\pm S D$ & $3.20 \pm 0.53$ & $3.25 \pm 0.56$ & 0.829 \\
\hline \multicolumn{4}{|l|}{ Hemiplegia, n(\%) } \\
\hline Left & $5 \rrbracket 33.33 \% \rrbracket$ & $7 \rrbracket 46.67 \% \rrbracket$ & 0.456 \\
\hline Right & $10 \rrbracket 66.76 \% \rrbracket$ & $8 \rrbracket 53.33 \% \rrbracket$ & \\
\hline Handedness & & & NS \\
\hline Left-handed & $1 \rrbracket 6.67 \% \rrbracket$ & 0 & \\
\hline Right-handed & $13 \rrbracket 86.67 \% \bigotimes$ & $15 \rrbracket 100 \% \rrbracket$ & \\
\hline Double-handed & $1 \otimes 6.67 \% \otimes$ & 0 & \\
\hline
\end{tabular}

Values are presented as means \pm standard deviations and percentages. Abbreviations: UL group: Usual exercise group, NS: not applicable.

\section{fNIRS results}

\section{Analysis of $\mathrm{O}_{2} \mathrm{Hb}$ changes based on $\mathrm{ROI}$ within-group and between-group comparisons}

In the Orthosis group, 1 case of ambidexterity and 1 case of left-handedness were excluded, leaving $\mathrm{O}_{2} \mathrm{Hb}$ data from 13 right-handed cases for analysis. In the UL group, 2 cases of incomplete or interfering data were removed, leaving 13 cases of right-handedness to analyze. Changes in the mean $\mathrm{O}_{2} \mathrm{Hb}$ 
concentrations in the PMC and SMC pre and post-intervention were compared between the two groups (concentration unit: $\times 10^{-6} \mu \mathrm{mmol} / \mathrm{l}$ ).

\section{Within-group comparisons}

In the Orthosis group, the mean $\mathrm{O}_{2} \mathrm{Hb}$ concentration for the ipsilateral PMC and contralateral PMC increased after intervention but not significantly $(p=0.178$ and $p=0.341)$. The mean $\mathrm{O}_{2} \mathrm{Hb}$ concentration for the ipsilateral SMC and contralateral SMC increased but not significantly $(p=0.881$ and $p=0.419)$.

Also in the Usual Care group, the mean $\mathrm{O}_{2} \mathrm{Hb}$ concentration for the ipsilateral PMC increased after intervention as well as the mean $\mathrm{O}_{2} \mathrm{Hb}$ concentration for the contralateral PMC but not significantly $(p=0.89$ and $p=0.98)$. The mean $\mathrm{O}_{2} \mathrm{Hb}$ concentration for the ipsilateral SMC increased after intervention, but the mean $\mathrm{O}_{2} \mathrm{Hb}$ concentration of the contralateral SMC decreased, there were no significant differences $(p=0.38$ and $p=0.65)$. (Table 2$)$

Table 2 Changes in the mean $\mathrm{O}_{2} \mathrm{Hb}$ concentrations in the PMC and SMC within -Group

\begin{tabular}{|c|c|c|c|c|c|c|}
\hline & \multicolumn{2}{|c|}{ Orthosis group } & \multirow{2}{*}{$\begin{array}{l}\text { Within-group } \\
p=0.178\end{array}$} & \multicolumn{2}{|c|}{ UL group } & \multirow{2}{*}{$\begin{array}{l}\text { Within- } \\
\text { group } \\
p=0.89\end{array}$} \\
\hline Ipsi-PMC & Pre & Post & & Pre & Post & \\
\hline & 1.9508 & 4.9154 & & 2.9365 & 3.0368 & \\
\hline \multirow[t]{2}{*}{ Contra-PMC } & Pre & Post & \multirow[t]{2}{*}{$p=0.341$} & Pre & Post & \multirow[t]{2}{*}{$p=0.98$} \\
\hline & 1.9280 & 2.9764 & & 2.4449 & 2.4641 & \\
\hline \multirow[t]{2}{*}{ Ipsi-SMC } & Pre & Post & \multirow[t]{2}{*}{$p=0.881$} & Pre & Post & \multirow[t]{2}{*}{$p=0.38$} \\
\hline & 2.5539 & 2.7165 & & 2.4221 & 3.4261 & \\
\hline \multirow[t]{2}{*}{ Contra-SMC } & Pre & Post & \multirow[t]{2}{*}{$p=0.419$} & Pre & Post & \multirow[t]{2}{*}{$p=0.65$} \\
\hline & 2.1985 & 2.7729 & & 3.1034 & 2.4224 & \\
\hline
\end{tabular}

Values are presented as means. Abbreviation: Ipsi-:Ipsilesional, Contra-:Contralesional, $\mathrm{O}_{2} \mathrm{Hb}$ :

Oxygenated hemoglobin .

\section{Between-group comparisons}

The between-group comparison showed no significant differences in mean $\mathrm{O}_{2} \mathrm{Hb}$ changes in the PMC and SMC between the two groups: the change in the ipsilateral PMC $(F=5.912, p=0.364)$; the change in the contralateral PMC $(F=1.315, p=0.302)$, the change in the ipsilateral SMC $(F=0.008, p=0.556)$, and the change in the contralateral SMC $(F=0.567, p=0.400)($ Table 3$)$ 
Table 3 Changes in the mean $\mathrm{O}_{2} \mathrm{Hb}$ concentrations in the PMC and SMC between-Group

\begin{tabular}{lllll} 
& & Orthosis group & UL group & Between-group \\
\hline Ipsi-PMC & Post-Pre & 2.9645 & 0.1002 & $\mathrm{p}=0.364$ \\
\hline Contra-PMC & Post-Pre & 1.0483 & 1.0029 & $\mathrm{p}=0.302$ \\
\hline Ipsi-SMC & Post-Pre & 0.1626 & 0.0191 & $\mathrm{p}=0.556$ \\
\hline Contra-SMC & Post-Pre & 0.5744 & -0.68105 & $\mathrm{p}=0.400$
\end{tabular}

Values are presented as means. Abbreviation: Ipsi-:Ipsilesional, Contra-:Contralesional, $\mathrm{O}_{2} \mathrm{Hb}$ :

Oxygenated hemoglobin .

\section{Analysis of LI}

\section{Within-group comparisons}

In the Orthosis group, the LI for the PMC decreased from positive to negative, indicating a shift in the cortical activation from the ipsilateral PMC to the contralateral PMC after intervention. The mean LI value of the SMC increased from negative to positive, suggesting a shift in the cortical activation from the contralateral SMC to the ipsilateral SMC after the intervention. In the Usual Care group, the mean LI value for the PMC decreased, suggesting a shift in cortical activation from the ipsilateral to the contralateral PMC. The mean $\mathrm{LI}$ value of the SMC increased from negative to positive, suggesting a shift in cortical activation from the contralateral SMC to the ipsilateral SMC after treatment (Table 4).

Table 4 The mean LI value of the PMC and SMC of two groups

\begin{tabular}{lllll}
\hline \multicolumn{3}{l}{ Orthosis group } & \multicolumn{2}{l}{ UL group } \\
\hline & Pre & Post & Pre & Post \\
\hline PMC & $0.53 \pm 1.06$ & $-0.32 \pm 0.69$ & $0.08 \pm 0.44$ & $0.03 \pm 0.67$ \\
\hline SMC & $-0.84 \pm 1.27$ & $0.10 \pm 0.88$ & $-0.18 \pm 0.54$ & $0.21 \pm 0.39$
\end{tabular}

\section{Between-group comparisons}

No significant differences in the change of $L I$ values for $\operatorname{SMC}(p=0.074)$, and no significant differences in the change of $\mathrm{LI}$ values for $\mathrm{PMC}(\mathrm{p}=0.087)$ between the two groups. 
All the persons with stroke $(n=30)$ completed all clinical scale tests. In this study, change scores of ARAT and FMA-Arm will be reported in the correlation analysis.

In the Orthosis group, a significant positive linear correlation was observed between the change of FMAarm scores and LI changes for PMC ( $\left.r=0.588, p^{*}=0.035\right)$ (Fig. 3 A), ARAT scores and LI changes for SMC $\left(r=0.554, p^{*}=0.0495\right)$ (Fig. $\left.3 \mathrm{D}\right)$. But no significant linear correlation was observed between the change of FMA-arm scores and LI changes for SMC $(r=-0.010, p=0.974)$ (Fig. $3 \mathrm{~B})$, the change of ARAT scores and LI changes for PMC ( $r=0.177, p=0.563)$ (Fig. $3 \mathrm{C})$.

In the Usual Care group, a significant negative linear correlation was observed between the change of FMA-arm scores and LI changes for SMC ( $\left.r=-0.705, p^{*}=0.007\right)$ (Fig. $\left.3 B^{\prime}\right)$. But no significant linear correlation was observed between changes of FMA-arm scores and $\mathrm{LI}$ changes for PMC $(r=0.327$, $p=0.276)$ (Fig. $\left.3 A^{\prime}\right)$, the changes of ARAT scores and LI changes for PMC $(r=-0.503, p=0.863)$ (Fig. $\left.3 C^{\prime}\right)$, the changes of ARAT scores and LI changes for SMC ( $r=0.068, p=0.825)$ (Fig. $\left.3 D^{\prime}\right)$.

\section{Discussion}

In this study, we analyzed the change of $\mathrm{O}_{2} \mathrm{Hb}$ in bilateral SMC, PMC by fNIRS during grip-release task in subacute persons with stroke following task-oriented training combined with and without a dynamic hand orthosis, no significant difference was between group. Similar shift of activation from the contralateral to the ipsilateral SMC, from the ipsilateral to contralateral PMC were observed in both groups. A significant positive correlation was only observed between the shift to contralateral PMC activation and change of FMA-arm score, between the shift to contralateral SMC and change of ARAT score in the Orthosis group. In the UL group, a negative correlation was only observed between the shift to ipsilateral SMC and changes of FMA score.

\section{Analysis of Oxygenated Hemoglobin Changes}

Many studies have shown that $\mathrm{O}_{2} \mathrm{Hb}$ was the most sensitive marker for changes in regional cerebral blood flow activity (31). Previous studies have suggested that the recovery of hand function after stroke was related to the activation of some cortical areas, such as the bilateral SMC, PMC, and cerebellum(33). In line with previous studies(34), our results indicate that the increased activation of the bilateral SMC and PMC has a compensatory role for both groups. A decrease in $\mathrm{O}_{2} \mathrm{Hb}$ concentration in contralesionally SMC in UL group may represent the reduced demand for task-related synaptic activity, suggesting that fewer neurons are required to complete the same action task as previous study ${ }^{(35)}$.However, no significant difference was found in activation between the two groups, most likely due to small sample size.

Based on these $\mathrm{O}_{2} \mathrm{Hb}$ result, in the future study, if a paired sample design is assumed to compare the differences of ipsilateral PMC within-group, to achieve $80 \%$ power of test, at alpha $=0.05,1$ - 
$\beta=0.8, \delta=1.96 \times 2.96=5.80$, a total of 562 sample size (two-tailed) in Orthosis group will achieve. If a group design is assumed to compare the differences of ipsilateral PMC between-group, to achieve $80 \%$ power of test, at alpha $=0.05,1-\beta=0.8, q 1=q 2=0.5, \delta=1.96 \times 2.86=5.61$, a total of 193 sample size(two-tailed) in each group will achieve. The sample size of further study could be based on this study.

\section{Analysis of Lateralization Index (LI)}

Studies have shown that the LI can be used to evaluate the recovery of motor function in persons with stroke(20) (36) (37). There is a similarity in cortical activation patterns during this grip-release task between two groups. Our results are compatible with some fMRI researches that the shift of SMC and PMC activation paralleled functional recovery of the hand (38).

Based on previous studies, PMC is involved in the purposeful modification and initiation of motion through connections with the brain stem, basal ganglia, cerebellum, and spinal cord(39).PMC activation may reflect the need for stabilizing proximal limbs and related to improved control of upper-limb performance(40). This activation of the contralateral brain area provides recruitment of additional neural resources due to the increased demands of the damaged motor system $(41,42)$.

SMC is an integral part of basic motor network associated with upper-limb recovery, our study supports this enhanced compensation in ipsilateral SMC(43) (38), this result was consistent with most studies that report a recovery of motor function (21) (40). These results reflect the reorganization of brain areas, however, the role of ipsilateral SMC or contralateral SMC has been controversial, opposite results could be seen in some studies $(36,40)$.

There are some reasons to explain these results of no difference $\triangle$ Firstly, according to the timeline of stroke recovery, the first week until the first month post-stroke is a critical time for neural plasticity and should be a target for recovery trials(6). However, if the persons were enrolled within 4 weeks since stroke, they may only have arm synergies, without hand grasping movement. More suitable motor task should be considered for fNIRS in the early subacute period. Secondly, only two test points and 4-weeks observation time may be insufficient. Applying repeated measurements at series of time points that start early and continue into the chronic phase in larger sample persons may help to establish understanding of recovery mechanism. Informed by series of time points data, new treatments would have a higher likelihood of being identified a true treatment effect. Third, the complexity of the task and motor intensity may affect the results(44-47). Cortical hemodynamic response to simple or complex motor stimuli differ to duration and intensity(46) (48)『longer duration and higher intensity may lead to feasible results of group-level differences.

\section{Relationship between change of LI value and clinical test outcomes}


Some findings pointed that after received multidisciplinary rehabilitation therapy, a negative association between changes of FMA score and the shift to ipsilateral M1 (primary motor area), a positive association between changes of ARAT score and the shift to contralateral M1 could be seen(43).Our study of UL group is consisted with these findings (Fig. 3,B'),but no similar trend could be seen in Orthosis group(Fig. 3,B). One possible explanation could be the compensatory contribution of ipsilateral M1 to motor recovery in UL group(40).Our study of Orthosis group is also consisted with Riecker's findings(43) (D), which means the shift to contralateral SMC activation plays on important role in the increase of ARAT score in Orthosis group. In a consistent functional magnetic imaging (fMRI) study performed by Bütefisch(49), the contralateral cortex was also shown to play an important role in functional recovery.

According to a systematic literature review (50), FMA was related to multi-joint associated movement, ARAT was related to hand movements combined with synergistic movement. Any shift in brain activity to the ipsilateral hemisphere correlates with improvement in ARAT, but not correlates with improvement in FMA. In our study, there was no significant correlation between LI changes for PMC and ARAT score in any of the group. ( Fig. $3 \mathrm{C}, \mathrm{C}^{\prime}$ ). Due to the great individual difference, the significant difference of correlation analysis in two groups may be affected. This result may indicate that the relationship between the lateralization shift of PMC and ARAT was not a sensitive indicator to show the recovery mechanism in the two group.

In the Orthosis group, a positive correlation was between changes of FMA-arm score and the shift to contralateral PMC (Fig. $3 \mathrm{~A}$ ), this result suggested that the shift to contralateral PMC activation which was related to the preparation of movement for control and posture was significant correlative to the upper limb motor function in the Orthosis group, but no significant correlation in the UL group(Fig. $3 A^{\prime}$ ). However, a negative correlation was in Shoji's small sample study(37), individual differences and small sample should be considered when interpreting these results.

\section{Limitations.}

There are several limitations of this study. Because of limited samples, larger variability in motor recovery could affect the interpretation of the results. Besides,

related to inclusion criteria and peculiarity of recruiting center, the time 14-90 days baseline with a mean day approximately 50-day is a bit late to make use of the therapeutic time window of approximately 20 days (4 weeks) for max influence on neuroplasticity by rehabilitative training. Lack of repeated measurements at each week is another limitation. In the further fNIRS analysis and study, other outcome measures, such as peak and time-to-peak should be concerned, $\mathrm{O}_{2} \mathrm{Hb}$ and $\mathrm{LI}$ values for $\mathrm{ROI}$ in different hemispheric dominance (51) subgroups may have more interesting results.

\section{Conclusions}


In this pilot study, after unilateral task-oriented training with and without an orthosis in subacute stroke, there was no significant difference in $\mathrm{O}_{2} \mathrm{Hb}$ concentrations change冈the shift of $\mathrm{LI}$ for SMC and PMC.The associations with FMA-arm, ARAT and the activation shift of ROI was different and moderate, possibly indicating the usefulness with fNIRS. Sufficient samples, intervention time, and different rehabilitation intensity should be considered to obtain strong evidence in the future studies.

\section{Declarations}

\section{Acknowledgements}

We are grateful to all persons who contributed to this study. We would like to say a special thanks to Hitachi Medical Corporation for providing us with the use of the machine and operation training.

\section{Authors' contributions}

C.J.Z.L.is the first author. T.Z.is the corresponding author. Y.W. and C.J.Z.L. implemented, enrolled participants and collected data. T.Z., B.L.,X.X.D designed the experiment. F.B.H.as the occupational therapy guide and explained fNIRS methodology. H.J.Z. as the clinical scale tester, Y.L.W. performed statistical analysis. All authors discussed and approved the final manuscript. C.J.Z.L. wrote the paper.

\section{Funding}

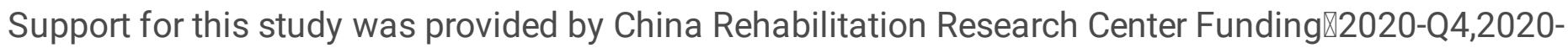
10,2019zx-01区and Sunnaastiftelsen.

\section{Availability of data and materials}

The datasets used in the study are available from the corresponding author on reasonable request.

\section{Declarations}

\section{Ethics approval and consent to participate}

The study was approved by the Regional Committee for Medical and Health Research Ethics (No.2017/1915 REK sør-øst D) and the China Rehabilitation Research Center Ethics Committee (No.2019112-1). Trial registration: Registered on January 11, 2018 at ClinicalTrials.gov 
(NCT03396939). https://clinicaltrials.gov/ct2/home .All participants provided written informed consent prior to enrolling in the study.

\section{Consent for publication}

No identifiable information is contained in this paper.

\section{Conflicting Interests}

The authors declared no potential conflicts of interest with respect to the research, authorship, and/or publication of this article.

\section{Author details}

1 School of Rehabilitation, Capital Medical University, Beijing, China.2 Department of Neurological Rehabilitation, Beijing Bo'ai Hospital, China Rehabilitation Research Center, Beijing, China.3 Department of Research, Sunnaas Rehabilitation Hospital, Norway.4 Institute of Clinical Medicine, Faculty of Medicine, University of Oslo, Norway. 5 Department of Physiotherapy, Faculty of Health Science, Oslo Metropolitan University, Oslo, Norway.6 Department of Occupational therapy, Beijing Bo'ai Hospital, China Rehabilitation Research Center, Beijing, China

\section{Abbreviations}

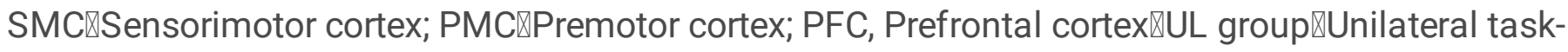

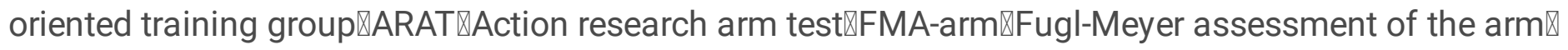

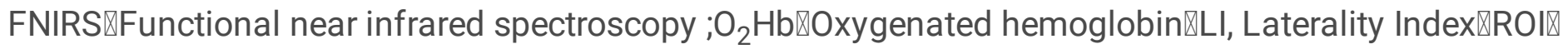
Region of interest\Ipsi-hemisphere邓Ipsilesional hemisphere『Contra-hemisphere『Contralesional hemisphere.

\section{References}

1. Murray CJ, Barber RM, Foreman KJ, Abbasoglu Ozgoren A, Abd-Allah F, Abera SF, et al. Global, regional, and national disability-adjusted life years (DALYs) for 306 diseases and injuries and healthy life expectancy (HALE) for 188 countries, 1990-2013: quantifying the epidemiological transition. Lancet.2015;386(10009):2145-91. 
2. Lozano R, Naghavi M, Foreman K, Lim S, Shibuya K, Aboyans V, et al. Global and regional mortality from 235 causes of death for 20 age groups in 1990 and 2010: a systematic analysis for the Global Burden of Disease Study 2010. Lancet.2012;380(9859):2095-128.

3. Lawrence ES, Coshall C, Dundas R, Stewart J, Rudd AG, Howard R, et al. Estimates of the prevalence of acute stroke impairments and disability in a multiethnic population.Stroke. 2001;32(6):1279-84.

4. Nijland RH, van Wegen EE, Harmeling-van der Wel BC, Kwakkel G. Presence of finger extension and shoulder abduction within 72 hours after stroke predicts functional recovery: early prediction of functional outcome after stroke: the EPOS cohort study. Stroke. 2010;41(4):745-50.

5. van Lieshout ECC, Boonzaier J, Pel AJ, van Heijningen CL, Vink JJ, Visser-Meily JMA, et al. Translational Value of Skilled Reaching Assessment in Clinical and Preclinical Studies on Motor Recovery After Stroke. Neurorehabil Neural Repair. 2021;35(5):457-67.

6. Bernhardt J, Hayward KS, Kwakkel G, Ward NS, Wolf SL, Borschmann K, et al. Agreed definitions and a shared vision for new standards in stroke recovery research: The Stroke Recovery and Rehabilitation Roundtable taskforce. Int J Stroke.2017;12(5):444-50.

7. Cramer SC. Treatments to Promote Neural Repair after Stroke. J stroke. 2018;20(1):57-70.

8. Cassidy JM, Cramer SC. Spontaneous and Therapeutic-Induced Mechanisms of Functional Recovery After Stroke. Transl Stroke Res. 2017;8(1):33-46.

9. Kitago T, Krakauer JW. Motor learning principles for neurorehabilitation. Handb Clin Neuro. 2013;110:93-103.

10. Pollock A, Farmer SE, Brady MC, Langhorne P, Mead GE, Mehrholz J, et al. Interventions for improving upper limb function after stroke.Cochrane database Syst Rev.2014(11):Cd010820.

11. Hughes AM, Burridge JH, Demain SH, Ellis-Hill C, Meagher C, Tedesco-Triccas L, et al. Translation of evidence-based Assistive Technologies into stroke rehabilitation: users' perceptions of the barriers and opportunities.BMC Health Serv Res. 2014;14:124.

12. de Araújo RC, Rocha DN, Pitangui AC, Pinotti M. The influence of dynamic orthosis training on upper extremity function after stroke: a pilot study. J Healthc Eng. 2014;5(1):55-66.

13. Franck JA, Smeets R, Seelen HAM. Evaluation of a functional hand orthosis combined with electrical stimulation adjunct to arm-hand rehabilitation in subacute stroke patients with a severely to moderately affected hand function. Disabil Rehabil. 2019;41(10):1160-8.

14. Mehrholz J, Pohl M, Platz T, Kugler J, Elsner B. Electromechanical and robot-assisted arm training for improving activities of daily living, arm function, and arm muscle strength after stroke. Cochrane database Syst Rev.2018;9(9):Cd006876.

15. Wong Y, Ada L, Wang R, Månum G, Langhammer B. Self-administered, home-based, upper limb practice in stroke patients: A systematic review. J Rehabil Med. 2020;52(10):jrm00118.

16. Leff DR, Orihuela-Espina F, Elwell CE, Athanasiou T, Delpy DT, Darzi AW, et al. Assessment of the cerebral cortex during motor task behaviours in adults: a systematic review of functional near infrared spectroscopy (fNIRS) studies. Neuroimage. 2011;54(4):2922-36. 
17. Gramigna V, Pellegrino G, Cerasa A, Cutini S, Vasta R, Olivadese G, et al. Near-Infrared Spectroscopy in Gait Disorders: Is It Time to Begin? Neurorehabil Neural Repair. 2017;31(5):402-12.

18. Al-Yahya E, Johansen-Berg H, Kischka U, Zarei M, Cockburn J, Dawes H. Prefrontal Cortex Activation While Walking Under Dual-Task Conditions in Stroke: A Multimodal Imaging Study. Neurorehabil Neural Repair. 2016;30(6):591-9.

19. Mihara M, Miyai I. Review of functional near-infrared spectroscopy in neurorehabilitation. Neurophotonics. 2016;3(3):031414.

20. Delorme M, Vergotte G, Perrey S, Froger J, Laffont I. Time course of sensorimotor cortex reorganization during upper extremity task accompanying motor recovery early after stroke: An fNIRS study. Restor Neurol Neurosci. 2019;37(3):207-18.

21. Lim SB, Eng JJ. Increased Sensorimotor Cortex Activation With Decreased Motor Performance During Functional Upper Extremity Tasks Poststroke. J Neurol Phys Ther. 2019;43(3):141-50.

22. Ferrari M, Quaresima V. A brief review on the history of human functional near-infrared spectroscopy (fNIRS) development and fields of application. Neuroimage. 2012;63(2):921-35.

23. Jeppesen J, Beniczky S, Johansen P, Sidenius P, Fuglsang-Frederiksen A. Exploring the capability of wireless near infrared spectroscopy as a portable seizure detection device for epilepsy patients. Seizure. 2015;26:43-8.

24. Arya KN, Verma R, Garg RK, Sharma VP, Agarwal M, Aggarwal GG. Meaningful task-specific training (MTST) for stroke rehabilitation: a randomized controlled trial.Top Stroke Rehabil. 2012;19(3):193211.

25. Teo WP, Goodwill AM, Hendy AM, Muthalib M, Macpherson H. Sensory manipulation results in increased dorsolateral prefrontal cortex activation during static postural balance in sedentary older adults: An fNIRS study. Brain Behav. 2018;8(10):e01109.

26. Tsuzuki D, Dan I. Spatial registration for functional near-infrared spectroscopy: from channel position on the scalp to cortical location in individual and group analyses. Neuroimage.2014;85 Pt 1:92-103.

27. Huppert TJ, Diamond SG, Franceschini MA, Boas DA. HomER: a review of time-series analysis methods for near-infrared spectroscopy of the brain. Applied optics. 2009;48(10):D280-98.

28. Franceschini MA, Joseph DK, Huppert TJ, Diamond SG, Boas DA. Diffuse optical imaging of the whole head. J Biomed Opt. 2006;11(5):054007.

29. Julien C. The enigma of Mayer waves: Facts and models. Cardiovas Res.2006;70(1):12-21.

30. Lloyd-Fox S, Begus K, Halliday D, Pirazzoli L, Blasi A, Papademetriou M, et al. Cortical specialisation to social stimuli from the first days to the second year of life: A rural Gambian cohort. Dev Cogn Neurosci. 2017;25:92-104.

31. Iso N, Moriuchi T, Fujiwara K, Matsuo M, Mitsunaga W, Hasegawa T, et al. Hemodynamic Signal Changes During Motor Imagery Task Performance Are Associated With the Degree of Motor Task Learning. Front Hum Neurosci. 2021;15:603069. 
32. Chou PH, Lin WH, Li WR, Huang CM, Sun CW. Reduced language lateralization in first episode schizophrenia: A near infrared spectroscopy study. Prog Neuropsychopharmacol Biol Psychiatry. 2017;78:96-104.

33. Marshall RS, Perera GM, Lazar RM, Krakauer JW, Constantine RC, DeLaPaz RL. Evolution of cortical activation during recovery from corticospinal tract infarction. Stroke. 2000;31(3):656-61.

34. Fujimoto H, Mihara M, Hattori N, Hatakenaka M, Kawano T, Yagura H, et al. Cortical changes underlying balance recovery in patients with hemiplegic stroke. Neuroimage. 2014;85 Pt 1:547 - 54.

35. Park SW, Butler AJ, Cavalheiro V, Alberts JL, Wolf SL. Changes in serial optical topography and TMS during task performance after constraint-induced movement therapy in stroke: a case study. Neurorehabil Neural Repair. 2004;18(2):95-105.

36. Favre I, Zeffiro TA, Detante O, Krainik A, Hommel M, Jaillard A. Upper limb recovery after stroke is associated with ipsilesional primary motor cortical activity: a meta-analysis. Stroke. 2014;45(4):1077-83.

37. Kinoshita S, Tamashiro H, Okamoto T, Urushidani N, Abo M. Association between imbalance of cortical brain activity and successful motor recovery in sub-acute stroke patients with upper limb hemiparesis: a functional near-infrared spectroscopy study. Neuroreport. 2019;30(12):822-7.

38. Pelicioni MC, Novaes MM, Peres AS, Lino de Souza AA, Minelli C, Fabio SR, et al. Functional versus Nonfunctional Rehabilitation in Chronic Ischemic Stroke: Evidences from a Randomized Functional MRI Study. Neural plasticity. 2016;2016:6353218.

39. Bajaj S, Drake D, Butler AJ, Dhamala M. Oscillatory motor network activity during rest and movement: an fNIRS study. Front Syst Neurosci.2014;8:13.

40. Miyai I, Yagura H, Hatakenaka M, Oda I, Konishi I, Kubota K. Longitudinal optical imaging study for locomotor recovery after stroke. Stroke. 2003;34(12):2866-70.

41. Buetefisch CM. Role of the Contralesional Hemisphere in Post-Stroke Recovery of Upper Extremity Motor Function. Front Neurol. 2015;6:214.

42. Rehme AK, Fink GR, von Cramon DY, Grefkes C. The role of the contralesional motor cortex for motor recovery in the early days after stroke assessed with longitudinal FMRI. Cerebral cortex. 2011;21(4):756-68.

43. Riecker A, Gröschel K, Ackermann H, Schnaudigel S, Kassubek J, Kastrup A. The role of the unaffected hemisphere in motor recovery after stroke. Hum Brain Mapp. 2010;31(7):1017-29.

44. Issard C, Gervain J. Variability of the hemodynamic response in infants: Influence of experimental design and stimulus complexity. Dev Cogn Neurosci.2018;33:182-93.

45. Holper L, Wolf M. Single-trial classification of motor imagery differing in task complexity: a functional near-infrared spectroscopy study. J neuroeng Rehabil. 2011;8:34.

46. Bendahan D, Chatel B, Jue T. Comparative NMR and NIRS analysis of oxygen-dependent metabolism in exercising finger flexor muscles. Am J Physiol Regul Integr Comp Physiol. 2017; 313(6): R740-53. 
47. Byun K, Hyodo K, Suwabe K, Kujach S, Kato M, Soya H. Possible influences of exercise-intensitydependent increases in non-cortical hemodynamic variables on NIRS-based neuroimaging analysis during cognitive tasks:Technical note. J Exerc Nutrition Biochem.2014;18(4):327-32.

48. Carius D, Andrä C, Clauß M, Ragert P, Bunk M, Mehnert J. Hemodynamic Response Alteration As a Function of Task Complexity and Expertise-An fNIRS Study in Jugglers. Front Hum Neurosci. 2016;10:126.

49. Bütefisch CM, Kleiser R, Körber B, Müller K, Wittsack HJ, Hömberg V, et al. Recruitment of contralesional motor cortex in stroke patients with recovery of hand function. Neurology. 2005;64(6):1067-9.

50. Santisteban L, Térémetz M, Bleton JP, Baron JC, Maier MA, Lindberg PG. Upper Limb Outcome Measures Used in Stroke Rehabilitation Studies: A Systematic Literature Review. PloS one. 2016;11(5):e0154792.

51. Liew SL, Garrison KA, Ito KL, Heydari P, Sobhani M, Werner J, et al. Laterality of Poststroke Cortical Motor Activity during Action Observation Is Related to Hemispheric Dominance. Neural Plast.2018;2018:3524960.

\section{Figures}




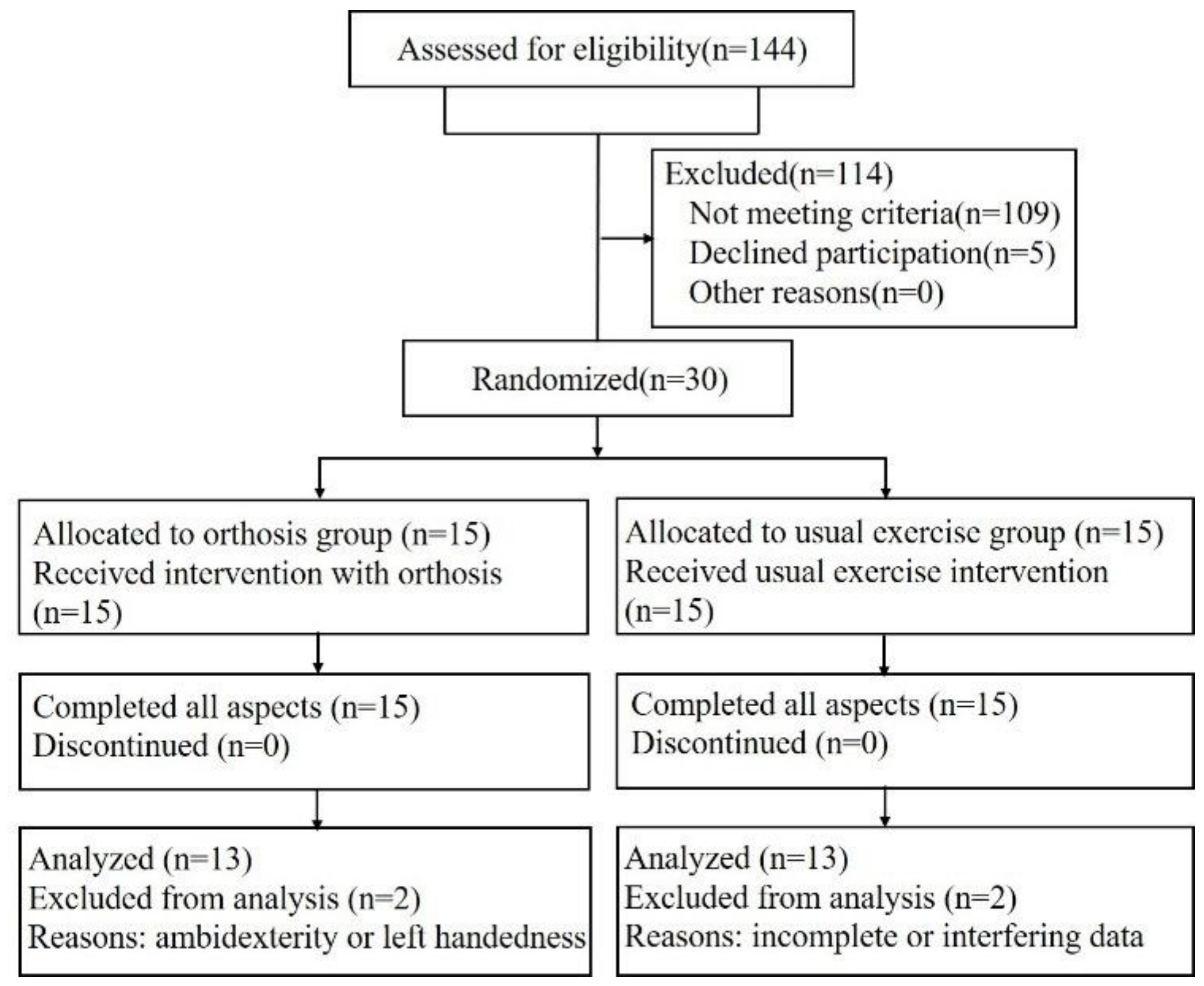

Figure 1

Flowchart of steps 

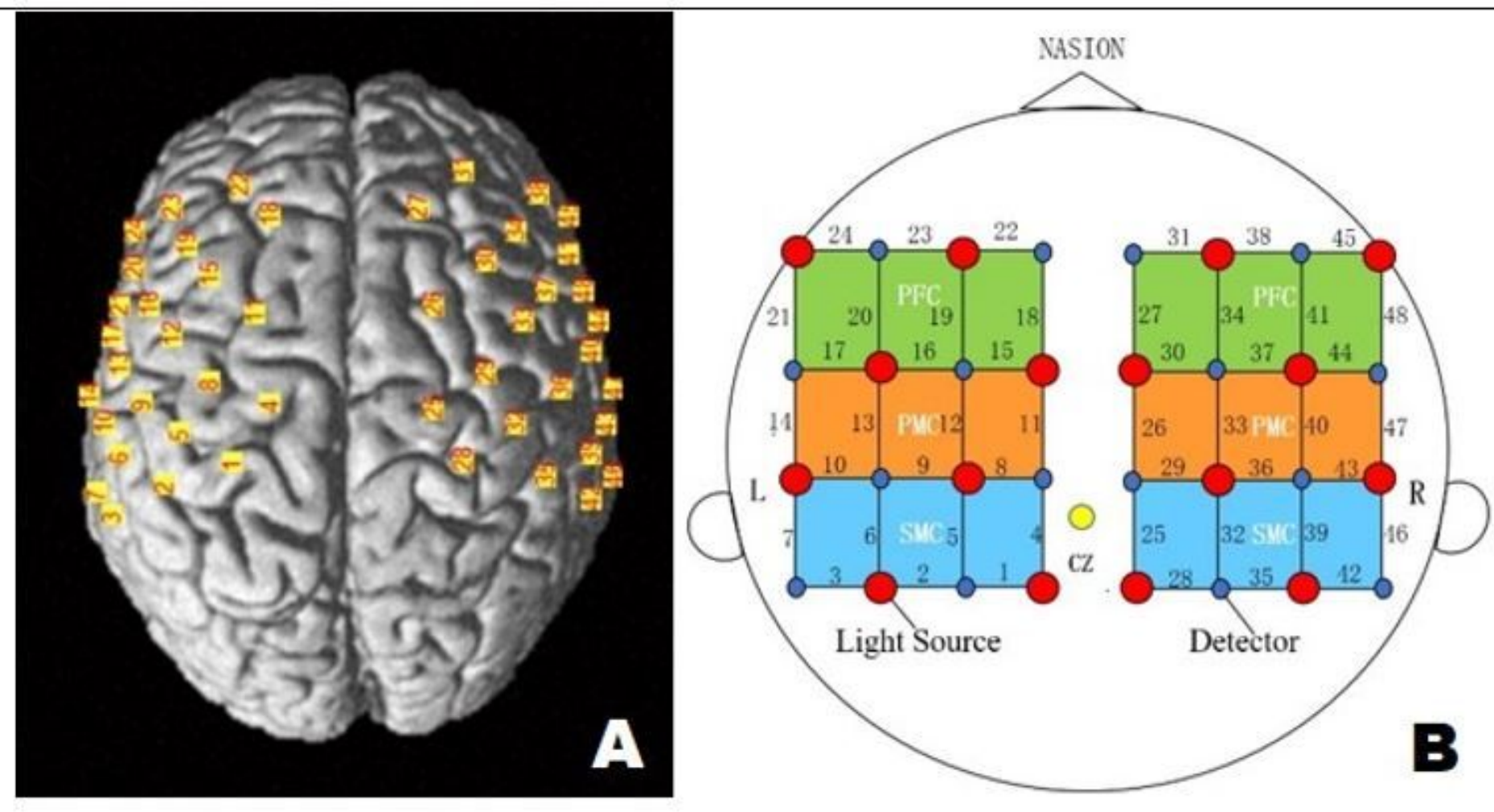

Figure 2

Experimental setting of the optodes A. 3D location of the optodes exposed to the brain surface of a standard brain. A total of 32 optodes. B. The channels covering the SMC, PMC and PFC, comprising 8 light source fibers (red) and 8 detectors (blue), were arranged on the scalp to enable 48 channel measurements. The $\mathrm{Cz}$ position of the international $10-20$ system was the marker for ensuring the replicable placement of the optodes.

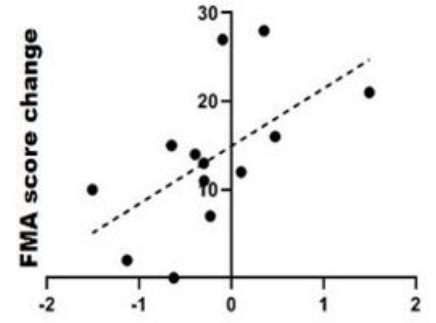

Orthosis Group PMC-LI change A

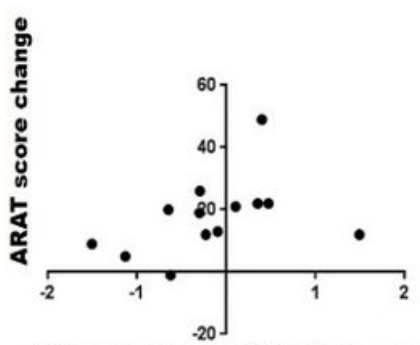

Orthosis Group PMC-LI change C

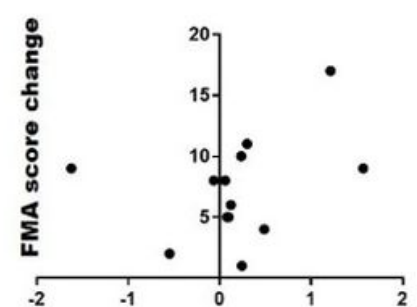

UL Group PMC-LI change A'

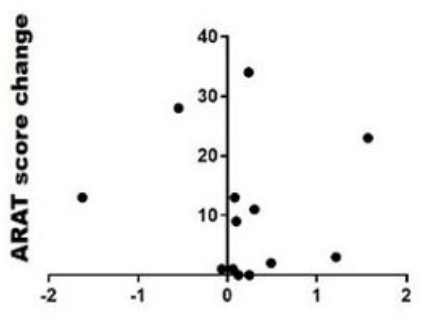

UL Group PMC-LI change

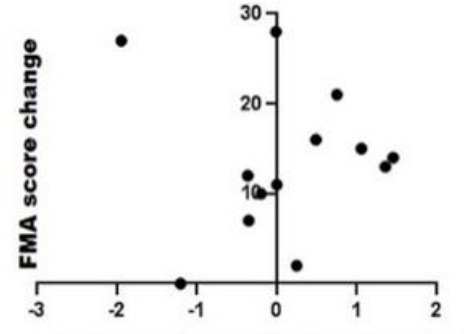

Orthosis Group SMC-LI change B

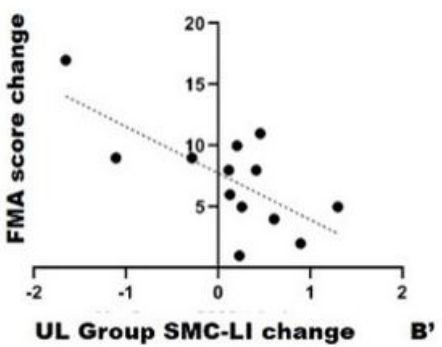

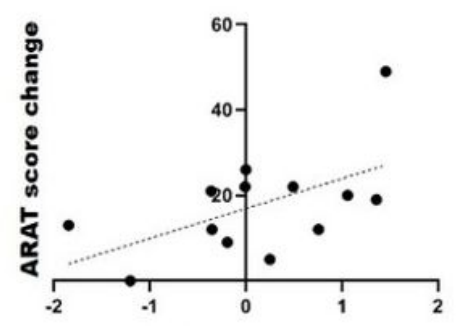

Orthosis Group SMC-LI change D

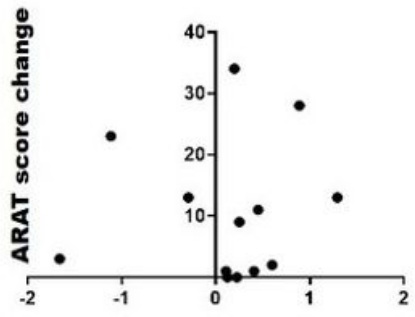

UL Group SMC-LI change D'

Figure 3 
Correlation for the paretic arm between changes for PMC, SMC and clinical tests in two groups A A' Correlation between laterality index changes for PMC囚PMC-LIXand FMA-arm scores in the Orthosis group $(A)$ and UL group ( $\left.A^{\prime}\right)$; B B' Correlation between laterality index changes for SMC囚SMC-LI冈and FMA-arm scores in the Orthosis group (B) and UL group $\left(B^{\prime}\right) ; C C^{\prime}$ Correlation between laterality index changes for

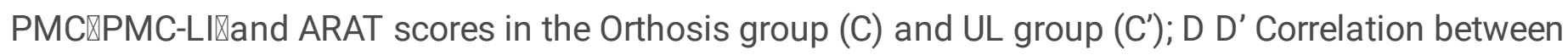
laterality index changes for SMC $\triangle S M C$-LI区and ARAT scores in the Orthosis group(D) and UL group ( $\left.D^{\prime}\right)$; A point (black circle) represents a change for a single patient measurement. The dotted line indicates that there is a significant linear correlation.

\section{Supplementary Files}

This is a list of supplementary files associated with this preprint. Click to download.

- CONSORTextensionforPilotChecklist.doc 